\title{
ENDOGENOUS STRESS RESPONSE ON PENTATONIC MUSIC IN VERY LOW BIRTHWEIGHT
} INFANTS

\author{
D. Schwilling ${ }^{1}$, M. Vogeser ${ }^{2}$, F. Kirchhoff ${ }^{2}$, F. Schwaiblmair ${ }^{1}$, A. Schulze ${ }^{1}$, A.W. Flemmer ${ }^{1}$ \\ ${ }^{1}$ Division of Neonatology, Ludwig-Maximilian-University Munich-Grosshadern, ${ }^{2}$ Institute of Clinical \\ Chemistry, Ludwig-Maximilian-University Munich-Grosshadern, Munich, Germany
}

Background: Music exposition of prematures has previously been reported to reduce pain, improve vital signs and result in overall non-specific "well-being". Valid data on endogenous stress hormone response to music, determined by salivary cortisol levels, are scarce.

Objective: The aim of this study was to test the effect of live pentatonic music on the ambient stress level of very low birthweight infants.

Methods: Stable infants (birthweight 500 - 1500 g, mean age: 22 days, $n=16$ ) were exposed to 15 minutes of live harp music on 3 consecutive days, at the same time of the day. Music was identically played on a kantele. Saliva was collected prior to music exposition and at defined time points after music. Salivary cortisol and cortisone levels were measured by liquid-chromatography-tandem-mass-spectrometry and salivary cortisol additionally by ELISA $(\mathrm{n}=10)$.

Stress levels were additionally assessed by recordings of heart rate, respiratory rate and oxygen saturation. Pain level was determined by Bernese-Pain-Scale-for-Neonates (BSN).

Results: Salivary cortisol decreased from baseline to 25 minutes and 4 hours after intervention on all 3 days $(\mathrm{p}<0.05$ on day 1 and 3$)$. Apneas, the number of desaturations as well as BSN levels improved significantly on all 3 days $(\mathrm{p}<0.05)$.

Conclusion: Our findings indicate that pentatonic music exposition can decrease stress hormone levels of preterm neonates in a NICU environment. These are the first data regarding the effect of music exposition on stress hormone levels in a sufficient cohort of VLBW infants supporting a positive effect of music on these infants. 